INPLASY

PROTOCOL

To cite: Xu et al. The Value of Urinary Gonadotropins in the Diagnosis of Central

Precocious Puberty: A MetaAnalysis. Inplasy protocol 2021120076. doi:

10.37766/inplasy2021.12.0076

Received: 16 December 2021

Published: 16 December 2021

Corresponding author:

Tao Liu

liutao1241@ncu.edu.cn

Author Affiliation:

Nanchang University

Support: No financial support.

Review Stage at time of this submission: Completed but not published.

Conflicts of interest:

None declared.

\section{The Value of Urinary Gonadotropins in the Diagnosis of Central Precocious Puberty: A Meta-Analysis}

Xu, D1; Zhou, XY2; Wang, JF3; Cao, X4; Liu, T5.

Review question / Objective: Precocious puberty is defined as the onset of secondary sexual characteristics before the age of 8 years in girls and 9 years in boys. It can be differentiated into central precocious puberty (CPP) and peripheral precocious puberty, and it is more common in girls than in boys. CPP may result in a decreased final adult height, an early age at menarche, and psychological and health problems in adulthood. Gonadotropin-releasing hormone (GnRH) GnRH stimulation test has been indispensable in the diagnosis of CPP. GnRH stimulation test is not only invasive, time-consuming and expensive, but also sometimes difficult to have patients cooperate. Nocturnal urinary LH and FSH can represent gonadotropin excretion in children with normal and early puberty. And urinary sample collection and evaluation are more convenient, more acceptable, cheaper, and noninvasive. This meta-analysis aims to assess the value of first-voided urinary luteinizing hormone (LH) and the ratio of urinary luteinizing hormone and follicle-stimulating hormone (FSH) in the diagnosis of female CPP and to compare the accuracy between urinary gonadotropins and serum GnRHstimulated gonadotropins.

INPLASY registration number: This protocol was registered with the International Platform of Registered Systematic Review and Meta-Analysis Protocols (INPLASY) on 16 December 2021 and was last updated on 16 December 2021 (registration number INPLASY2021120076).

\section{INTRODUCTION}

Review question / Objective: Precocious puberty is defined as the onset of secondary sexual characteristics before the age of 8 years in girls and 9 years in boys. It can be differentiated into central precocious puberty (CPP) and peripheral precocious puberty, and it is more common in girls than in boys. CPP may result in a decreased final adult height, an early age at menarche, and psychological and health problems in adulthood. Gonadotropinreleasing hormone (GnRH) GnRH 
stimulation test has been indispensable in the diagnosis of CPP. GnRH stimulation test is not only invasive, time-consuming and expensive, but also sometimes difficult to have patients cooperate. Nocturnal urinary LH and FSH can represent gonadotropin excretion in children with normal and early puberty. And urinary sample collection and evaluation are more convenient, more acceptable, cheaper, and noninvasive. This meta-analysis aims to assess the value of first-voided urinary luteinizing hormone (LH) and the ratio of urinary luteinizing hormone and folliclestimulating hormone (FSH) in the diagnosis of female CPP and to compare the accuracy between urinary gonadotropins and serum GnRH-stimulated gonadotropins.

Condition being studied: To date, many authors have found that urinary gonadotropin measurements are a potential alternative approach for the diagnosis of CPP. However, this remains a controversial issue as there are no unified standards and no evidence-based support for it. So, We searched six databases for relevant literature. In accordance with Preferred Reporting Items for Systematic Reviews and Meta-Analyses guidelines, we estimated sensitivity, specificity, area under the curve (AUC) of the summary receiver operator characteristic curve (SROC) and publication bias.

\section{METHODS}

Search strategy: Articles were searched for using a combination of keywords and free words. The keywords as search terms included "puberty, precocious", "urinary luteinizing hormone", and "urofollitropin". According to the PRISMA diagnostic test accuracy guidelines, the keywords regarding research methods included "sensitivity" and "accuracy". Each keyword and its free words were combined with "or". The different keywords were combined with "and". Search strategy are as follows: "Puberty, Precocious or Precocious Puberties or Puberties, Precocious or Pubertas Praecox or Praecox, Pubertas or Precocious Puberty or Precocious Puberty, Central or Central Precocious Puberties or Central Precocious Puberty or Precocious Puberties, Central or Puberties, Central Precocious or Puberty, Central Precocious Sexual Precocity or Precocities, Sexual or Precocity, Sexual or Sexual Precocities or Idiopathic Sexual Precocity or Idiopathic Sexual Precocities or Precocities, Idiopathic Sexual or Precocity, Idiopathic Sexual or Sexual Precocities, Idiopathic or Sexual Precocity, Idiopathic or Familial Precocious Puberty or Familial Precocious Puberties or Precocious Puberties, Familial or Precocious Puberty, Familial or Puberties, Familial Precocious or Puberty, Familial Precocious or Precocious Puberty, Male-Limited or Male-Limited Precocious Puberties or Male-Limited Precocious Puberty or Precocious Puberties, MaleLimited Puberties, Male-Limited Precocious or Puberty, Male-Limited Precocious or Precocious Puberty, Male Limited or Testotoxicosis" and "Urofollitropin or Follicle-Stimulating Hormone, Human Urine or Follicle Stimulating Hormone, Human Urine or Human FSH, Urinary or Urinary Human FSH or Fertinex or Metrodin or Neo Fertinorm or Metrodin HP or Metrodin High Purity or High Purity, Metrodin or Bravelle or Follitrin" and "luteinizing hormone or Hormone, Luteinizing or Lutropin LH (Luteinizing Hormone) or ICSH (Interstitial Cell Stimulating Hormone) or Interstitial Cell-Stimulating Hormone or Hormone, Interstitial Cell-Stimulating or Interstitial Cell Stimulating Hormone or Luteozyman or Luteoziman or urinary luteinizing hormone or urinary Hormone, Luteinizing or urinary Lutropin or urinary LH (urinary Luteinizing Hormone) or urinary ICSH (urinary Interstitial Cell Stimulating Hormone) or urinary Interstitial CellStimulating Hormone or urinary Hormone, Interstitial Cell-Stimulating or urinary Interstitial Cell Stimulating Hormone or urinary Luteozyman or urinary Luteoziman" and "Gonadotropins or Gonadotropin or Urinary Gonadotropins or Urinary Gonadotropin" and "sensitivity or sensitivity and specificity or (predictive AND value*) or predictive value of tests or accuracy". 
Participant or population: Participants were the girls, whose secondary sexual characteristics were presented before the age of 8 years, with accelerrated growth and mature bone age, and diagnosis of central prococious puberty after GnRH stimulation tests.

Intervention: Intervention is first-voided urinary GnRH evaluation, including ULH level and ULH:UFSHratio. First-voided urine is collected from subjects who were informed to empty their bladder before going to bed and to refrain from voiding until the next morning. First-voided urine is collected from subjects who were informed to empty their bladder before going to bed and to refrain from voiding until the next morning.

Comparator: The gold standard was a serum LH level $\geq 5 \mathrm{mIU} / \mathrm{L}$ or a serum LH:FSH ratio > 0.6 after $\mathbf{G n R H}$ stimulation test.

Study designs to be included: We conducted a meta-analysis of diagnostic test accuracy. This meta-analysis is reported following the Preferred Reporting Items for Systematic Reviews and Metaanalyses (PRISMA) guidelines.

Eligibility criteria: Eligibility criteria were asfollows: (1) Study was designed to be a diagnostic test study, and the results have beenpublished. (2) All the subjects in the studies were female with Tanner breast stage $\geq 2$, advanced bone age (BA) by $\geq 1$ year, and acceleratedgrowth. (3) Urinary and serum gonadotropin samples were collected on the same day for eachsubject. (4) All urinary samples came from the firstvoided urine. For reliable evaluation of urinary gonadotropins, all of the subjects must have been informed to empty their bladder before going to bed and to refrain from voiding until the nextmorning. (5) The gold standard was a serum LH level $\geq 5$ $\mathrm{mIU} / \mathrm{L}$ or a serum LH:FSH ratio $>0.6$.

Information sources: We searched the PubMed, Embase, Cochrane Library for clinical trials, Web of Science, China National Knowledge Infrastructure (CNKI), and Wanfang databases for relevant literature.

Main outcome(s): Both urinary LH level and urinary LH:FSH ratio are effective and available approaches for the diagnosis of true precocious puberty from an evidencebased view.

Data management: Microsoft Excel 2010 was used for data extraction. The relevant information that was required for this metaanalysis was extracted: the first author, publication year, nationality, gender, age, ethnicity, the number in the CPP group and the control group, the gold standard test results, and the sensitivity, specificity, true positive (TP), false positive (FP), true negative (TN), false negative (FN) and accuracy measures.

Quality assessment / Risk of bias analysis: Literature quality assessments were performed by two independent investigators. They independently extracted and incorporated the data. When disagreements arose, the investigators discussed the study until a consensus was reached. The assessment was performed based on the quality assessment of diagnostic accuracy studies(QUADAS-2).

Strategy of data synthesis: Meta Disc (version 1.40) was used to evaluate threshold effects and heterogeneity. We performed the Cochran-Q test to assess heterogeneity and used the inconsistency index (12 test) to assess the magnitude of heterogeneity among studies. If the $P$-value of the Cochran- $Q$ test was greater than 0.1 and the 12 value was lower than $50 \%$, there was no heterogeneity, and then a fixed effects model was performed. If the value of $\mathbf{P}<0.1$ or $12>50 \%$, it indicated that there was great heterogeneity, and then a random effects model was performed. The pooled sensitivity, pooled specificity, pooled diagnostic odds ratios (DORs), and pooled positive and negative likelihood ratios (LRs) were all calculated by Meta Disc, and the $P$-values and $95 \%$ confidence intervals (Cls) were calculated at the same time. Meanwhile, the area under the curve (AUC) of the summary receiver operator 
characteristic curve (SROC) were caldulated. Sensitivity evaluation and publication bias were performed with STATA (version 15.0). If >10 articles were included in this meta-analysis, we applied Deeks' funnel plot to assess the extent of potential publication bias. Otherwise, we used the Begg rank correlation and Egger tests to perform a publication bias analysis.

Subgroup analysis: There were Metaanalysis of urinary $\mathrm{LH}$ for the diagnosis of CPP and Meta-analysis of urinary LH:FSH ratio for the diagnosis of CPP.

Sensitivity analysis: The sensitivity analysis of urinary LH for the diagnosis of CPP illustrated that some of the included studies in this meta-analysis might have caused bias. Subsequent sensitivity analyses were conducted, and the results showed that the fourth record had the largest effect. The sensitivity analysis of urinary LH:FSH ratio for the diagnosis of CPP illustrated that two of the included studies in this meta-analysis might have caused bias. Moreover, we repeatedly reanalysed the data while excluding the relevant records one by one. After deleting the data from the first record, a threshold effect was found, so we did not further apply this analysis. As the data from the second record with an impact were eliminated, the results were not altered when this study was removed.

Language: English and Chinese.

Country(ies) involved: Korea, Denmark and China.

Keywords: Urinary gonadotropins, Gonadotropin-releasing hormone stimulation test, Central precocious puberty.

Contributions of each author:

Author 1 - Dan Xu designed the study, analyzed the data and participated in drafting manuscript.

Email: peanyhsu@163.com

Author 2 - Xueying Zhou participated in performing the study.

Email: 1907668425@qq.com
Author 3 - Junfei Wang participated in performing the study.

Email: 975128703@qq.com

Author 4 - Xi Cao participated in analyzing the data.

Email: 1114977049@qq.com

Author 5 - Tao Liu designed the study and revised the manuscript.

Email: liutao1241@ncu.edu.cn 